\title{
The design and optimization of novel elliptic cylindrical TSV and its temperature characterization
}

\author{
Wenbo Guan ${ }^{1}$, Hongliang $\mathrm{Lu}^{2}$, and Yuming Zhang ${ }^{1}$ \\ ${ }^{1}$ Xidian University \\ ${ }^{2}$ Affiliation not available
}

February 23, 2021

\begin{abstract}
Through Silicon Via (TSV) technology is a key technology to realize multi-layer chips and its structure model and transmission characteristics have attracted much attention. With the continuous reduction of chip size, higher requirements are put forward for the model and transmission characteristics of TSV. A novel elliptic cylindrical TSV structure model is proposed. The influence of axial length ratio, height, dielectric layer thickness and spacing on the transmission characteristics of TSV are further studied by HFSS software. The results show that the transmission of TSV is facilitated by the decrease of axial length ratio, the decrease of height, the increase of dielectric layer thickness and the increase of TSV spacing. The TSV structural parameter values are optimized by a single variable method. The optimized TSV structure is compared with the original TSV, traditional cylindrical and conical TSV and coaxial TSV. It is concluded that the elliptic cylindrical TSV structure has better transmission performance. The temperature characteristics of the elliptic cylindrical TSV are simulated. It is indicated that the transmission characteristics of the elliptic cylindrical TSV are poor at low frequency and better at high frequency when the temperature rises.
\end{abstract}

\section{Hosted file}

The design and optimization of novel elliptic cylindrical TSV and its temperature characterization.pdf available at https://authorea.com/users/397601/articles/510432-the-design-and-optimizationof-novel-elliptic-cylindrical-tsv-and-its-temperature-characterization 